\title{
Vivre avec des os de verre
}

\section{Therese Stutz Steigera ${ }^{a}$ Bérengère Rozier Aubry}

a Ancienne médecin, présidente Association Suisse Osteogenesis Imperfecta (SVOI-ASOI) 2011-2020; Vice-présidente ProRaris;

${ }^{b}$ Rhumatologue, spécialiste dans le soin aux patients avec maladies osseuses et locomotrices rares, CHUV, Lausanne

\begin{abstract}
En novembre dernier s'est tenue à Amsterdam une conférence internationale sur l'ostéogenèse imparfaite (OI), ou maladie des os de verre. Cet événement différait des congrès scientifiques habituels de par son originalité de s'adresser autant aux experts qu'aux personnes concernées par la maladie.
\end{abstract}

Parmi les 300 participants, la quantité et la variété des fauteuils roulants, entre autres moyens de transport similaires, ont montré qu'il y avait des patients OI de tous âges et avec des formes très différentes d'atteintes du tissu conjonctif. Sous la direction de Dagmar Mekking, la fondation Care4BrittleBones est l'initiatrice et l'organisatrice de l'événement. Elle a mis en place un excellent réseau des personnes atteintes et des experts de la maladie. Les participants de la Suisse étaient la Dre Bérengère Rozier Aubry, du Centre hospitalier universitaire vaudois (CHUV) à Lausanne, la Dre Therese Stutz Steiger, présidente de l'Association Suisse Osteogenesis Imperfecta (SVOI-ASOI), et Mark Steiger, proche aidant et père d'un jeune garçon atteint d'OI.

La conférence s'est déroulée dans le centre de conférence de la RAI d'Amsterdam, qui a d'abord paru surdimensionné. Les lieux ont permis aux personnes en fauteuil roulant et à celles avec des béquilles de ne jamais se sentir à l'étroit. Même pendant les pauses, les participants ont pu se servir de boissons et collations sans trop de temps d'attente. Grâce aux aménagements provisoires, la salle de conférence a été rendue particulièrement confortable pour les participants.

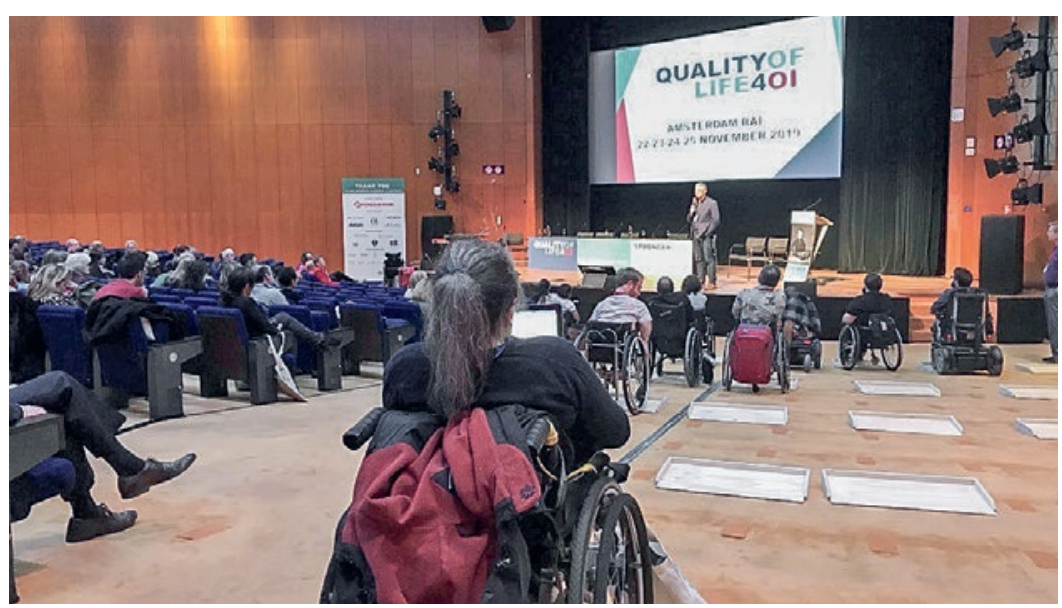

Grâce aux aménagements provisoires, la salle de conférence a été rendue particulièrement confortable pour les participants.

\section{A la fois patient et expert}

Les personnes touchées par l'OI, endossant parfois le double rôle de patient et d'expert, ont joué un rôle important dans le contenu des sessions. Les participants, soit patients, proches aidants, représentants des associations de patients, experts scientifiques et médicaux, ont été beaucoup plus actifs que lors des congrès conventionnels. A côté des principaux exposés ont été organisées des «Deep Diving Sessions» thématiques au cours desquelles les personnes concernées ont eu tout loisir de s'exprimer. Les sessions de présentation de posters ont également permis aux participants d'échanger et de faire plus ample connaissance. Le dîner de gala du congrès a aussi fait la part belle aux échanges interpersonnels, ponctué d'animations de jeunes participants aux talents artistiques étonnants.

Le point fort de ce congrès était de réunir toutes les personnes concernées par l'importance de mieux traiter l'OI chez les enfants et les adultes et de mettre en évidence les aspects qui affectent la vie et la qualité de vie des malades. Il s'agissait aussi de diffuser l'idée, autant auprès des personnes touchées que des professionnels, que la prise en charge et les efforts de tous peuvent être davantage abordés sous un angle de collaboration.

Une partie importante du programme a été consacrée aux sujets suivants:

- Thérapie médicamenteuse: des bisphosphonates à la thérapie par cellules souches

- Orthopédie: Road map to surgery for optimal patient satisfaction

- Diagnostic: tendances passées, actuelles et futures (focus génétique)

- Aspects psychosociaux: mesurer la qualité de vie

- Autonomisation: ce n'est qu'après ce processus, qui s'opère à différents niveaux, que les patients peuvent agir en tant que partenaires 


\section{Une maladie systémique}

La conférence a abordé d'autres points forts comme l'évolution du rôle du patient. Ingunn Westerheim, la présidente de l'Osteogenesis Imperfecta Federation Europe (OIFE), la faîtière européenne des organisations de patients atteints de OI, a tenu un exposé remarquable à ce sujet intitulé «The changing role of the patient». Elle a démontré que les besoins des patients en hôpital sont très différents de ceux des personnes vivant avec une maladie chronique. Un sujet hautement actuel était les rapports sur le réseau européen de référence ERN BOND, dont la Suisse est exclue n'étant pas membre de l'UE. La Grande-Bretagne a beaucoup contribué à ce réseau, dont le sort est incertain après le Brexit. La présentation du nouvel ensemble Key4OI a trouvé un large écho au sein du plénum. Key4OI est l'un des plus importants projets de collaboration dans le domaine de l'OI réunissant des experts de nombreux pays. Un quart d'entre eux sont atteints d'OI. Ces deux dernières années, ils ont déterminé les principaux problèmes de santé rencontrés par les personnes atteintes d'OI, tenant compte de tous les types d'OI. Ils ont discuté au niveau international de la manière dont les résultats des traitements devraient être mesurés. Médecins et scientifiques veulent ainsi acquérir de nouvelles connaissances sur l'efficacité du traitement de l'OI.

Une présentation sur l'OI en tant que maladie systémique, une approche relativement récente, a montré les différents effets de la maladie sur le corps: problèmes osseux et non osseux spécifiques, tels que les valves et les septums cardiaques, les vaisseaux, la cornée, le cartilage, les dents.

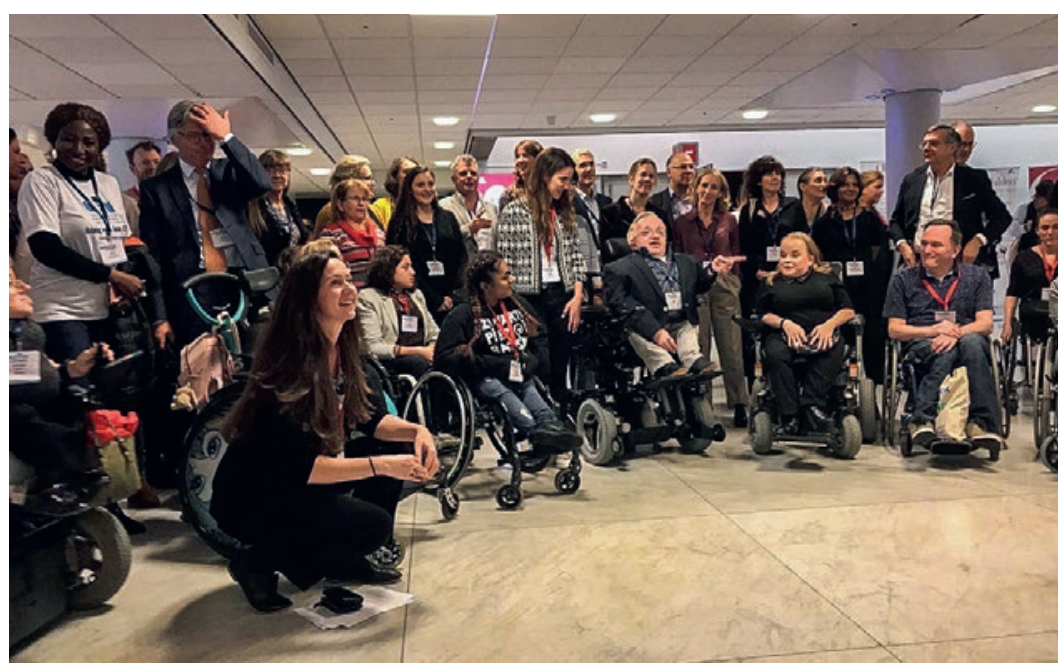

Parmi les 300 participants se trouvaient des patients Ol de tous âges et avec des formes très différentes d'atteintes du tissu conjonctif.

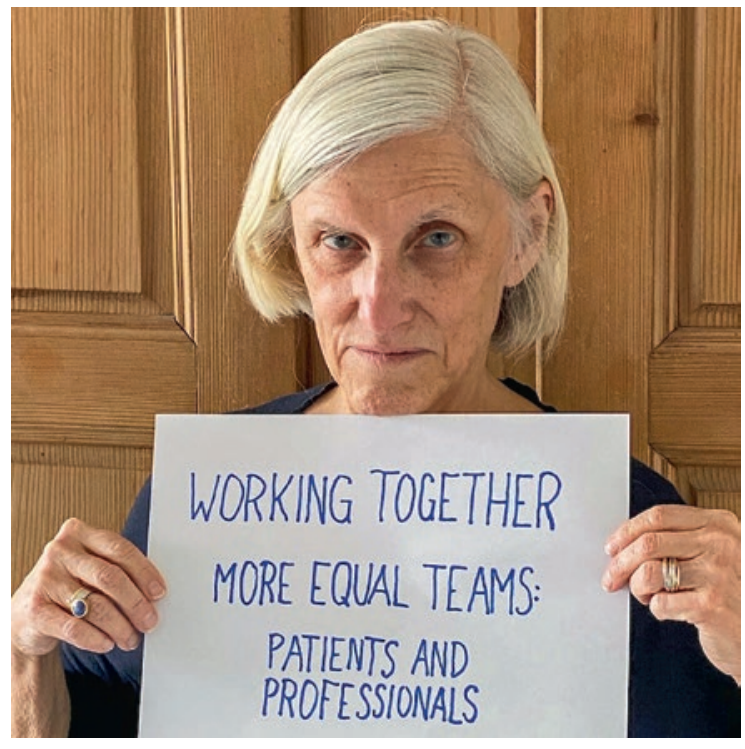

Therese Stutz Steiger est atteinte de la maladie des os de verre. Avec 60 personnes concernées de différents pays, elle a émis un vœu lors du "Wishbone Day» le 6 mai 2020.

\section{Poursuivre le dialogue}

Il est de plus en plus mis en évidence que les personnes souffrant d'OI et leurs familles ne parviennent pas toujours à faire face à la charge psychique. Certains patients affrontent l'anxiété et la dépression. Cette question a été abordée notamment lors des sessions thématiques «Vivre avec l'OI» et «Empowerment». Il reste encore beaucoup à faire dans ce domaine et Care4BrittleBones compte créer un groupe de travail international chargé d'explorer ces problématiques.

Ce congrès a permis de soigner le dialogue et l'échange entre les acteurs de la recherche, du monde clinique, des soins et des organisations de patients. Les contacts sur les réseaux sociaux se sont vu renforcer de retour à la maison. Reste à espérer que ces impulsions servent de base pour le prochain événement de cette ampleur, qu'elles soient approfondies et reflètent encore davantage le quotidien des personnes atteintes d'OI. De retour en Suisse, nous voulons renforcer le travail en réseaux et la collaboration avec les orthopédistes notamment.

\section{Remerciements}

Nous remercions Mark Steiger (proche aidant) et Cornelia Jungo (membre SVOI-ASOI) pour leur coopération.

\section{Crédits photos}

Therese Stutz Steiger, Mark Steiger

Pour plus d'informations sur l'ostéogenène imparfaite, rendezvous sur www.glasknochen.ch 